\title{
Pairing symmetry of superconducting graphene
}

\author{
F. M. D. Pellegrino ${ }^{1,2,3}$, G. G. N. Angilella ${ }^{1,2,3,4 a}$, and R. Pucci ${ }^{1,3}$ \\ ${ }^{1}$ Dipartimento di Fisica e Astronomia, Università di Catania, Via S. Sofia, 64, I-95123 Catania, Italy \\ ${ }^{2}$ Scuola Superiore di Catania, Via S. Nullo, 5/i, I-95123 Catania, Italy \\ ${ }^{3}$ CNISM, UdR Catania, I-95123 Catania, Italy \\ ${ }^{4}$ INFN, Sez. Catania, I-95123 Catania, Italy
}

the date of receipt and acceptance should be inserted later

\begin{abstract}
The possibility of intrinsic superconductivity in alkali-coated graphene monolayers has been recently suggested theoretically. Here, we derive the possible pairing symmetries of a carbon honeycomb lattice and discuss their phase diagram. We also evaluate the superconducting local density of states (LDOS) around an isolated impurity. This is directly related to scanning tunneling microscopy experiments, and may evidence the occurrence of unconventional superconductivity in graphene.
\end{abstract}

PACS. 81.05.ue Graphene - 74.25.Dw Superconductivity phase diagrams - 71.55.-i Impurity and defect levels

\section{Introduction}

Graphene is a one-atom-thick layer of graphite [1]. Due to its nearly ideal two-dimensional character and the linear, relativistic-like dispersion of its elementary excitations [2], graphene is an intriguing material, where correlation and reduced dimensionality may conspire in favor or against various electronic instabilities. Indeed, it has been proposed that an electron liquid in a honeycomb lattice can be characterized by several ordered states, depending on doping and on the electron-electron interaction [3]. Among these competing orders, superconductivity (SC) could be stabilized by either topological disorder [4] or the proximity to an electronic topological transition (ETT) [5]. In this case, the symmetry of the underlying lattice may allow for an unconventional structure of the SC order parameter, and possibly sizeable critical temperatures $T_{c}$, as is the case for the high- $T_{c}$ cuprates $6,7,8$.

The great interest in future graphene-based technology largely owes to the relatively high and easily tunable conductivity that can be realized in clean graphene samples. Electronic correlations and the possible occurrence of ordered phases are therefore often neglected. Recently, a superconducting current has been observed to propagate through a superconductor-normal-superconductor (SNS) Josephson junction, where the $\mathrm{N}$ region consisted of a graphene layer 9. This provided evidence of SC phase coherence in graphene single layers. Other carbonbased compounds are also known to sustain superconductivity, sometimes with fairly large critical temperatures $T_{c}$. These include (a) highly oriented pyrolytic graphite (HOPG) [10, (b) the graphite intercalated compounds (GIC) [11,12,13, which may be described as graphene sheets alternated by alkali layers, mainly acting as charge reservoirs, (c) quasi-one-dimensional carbon nanotubes, and (d) quasi-zero-dimensional alkali-doped fulleres, or fullerides [14]. In particular, recent $a b$ initio calculations support the idea that in-plane phonons may be responsible of SC in GIC [15, 16, 17, 18, 19,20. The quest for (quasi)intrinsic superconductivity in quasi-two-dimensional graphene is therefore well motivated, and several mechanisms have been proposed. These range from conventional, phonon-mediated superconductivity as in the GIC [15. $16,17,18,19,20,21$, to unconventional, electronic mechanisms, including particularly the resonating valence bond (RVB) mechanism [8,6. Moreover, it has been proposed 22 that SC may be realized in alkali-coated graphene single layers, where either electronic states belonging to the metallic bands get paired by means of graphene phonon modes, or graphene electrons couple via metallic plasmons 22 .

Here, after classifying the symmetries of a SC order parameter compatible with the honeycomb lattice of graphene, and discuss their stability and possible mixing as a function of doping and coupling strengths, we study the local density of states (LDOS) of SC graphene around an isolated impurity 23. In particular, we suggest that scanning tunneling microscopy (STM) measurements can distinguish among the various available SC symmetries in graphene, thereby evidencing the possible unconventional nature of the SC order parameter, as is the case for the superconducting state of high- $T_{c}$ cuprates [24,25], and possibly of their unconventional normal state [26, 27, 28, 


\section{Superconducting phase diagram}

We start by considering a model Hamiltonian $H=H_{0}+$ $H_{1}$, where $H_{0}=\sum_{\mathbf{k} \lambda} \xi_{\mathbf{k} \lambda} c_{\mathbf{k} \lambda}^{\dagger} c_{\mathbf{k} \lambda}$ describes the normal electron liquid, with $c_{\mathbf{k} \lambda}^{\dagger}\left(c_{\mathbf{k} \lambda}\right)$ a creation (annihilation) operator for a quasiparticle with wavevector $\mathbf{k}$ within the first Brillouin zone (1BZ) and band index $\lambda= \pm, \xi_{\mathbf{k} \lambda}=E_{\mathbf{k} \lambda}-\mu$ the tight-binding dispersion relation for band $\lambda$, measured with respect to the chemical potential $\mu$. Retaining hopping and overlap terms between nearest neighbor sites [29], one has $E_{\mathbf{k} \lambda}=\lambda t\left|\gamma_{\mathbf{k}}\right| /\left(1-\lambda s\left|\gamma_{\mathbf{k}}\right|\right)$, where $t=2.8 \mathrm{eV}$ and $s=0.07$ are the nearest neighbor hopping and overlap parameters, respectively [30], and $\gamma_{\mathbf{k}}=\sum_{\ell=1}^{3} e^{i \mathbf{k} \cdot \delta_{\ell}}$ is the usual (complex) structure factor in momentum space. Here, $\delta_{1}=a(1, \sqrt{3}) / 2, \delta_{2}=a(1,-\sqrt{3}) / 2, \delta_{3}=a(-1,0)$ are the vectors connecting nearest neighbor sites in real space, where $a=0.142 \mathrm{~nm}$ is the $\mathrm{C}-\mathrm{C}$ distance 2 .

As for the pairing Hamiltonian $H_{1}$, we restrict to onsite $\left(V_{o n}\right)$ and nearest-neighbor $\left(V_{n n}\right)$ interaction only. In terms of separate sets of creation and annihilation operators $a_{\mathbf{k} \sigma}^{\dagger}, a_{\mathbf{k} \sigma}\left(b_{\mathbf{k} \sigma}^{\dagger}, b_{\mathbf{k} \sigma}\right)$ for the $A(B)$ sublattices, along the singlet channel and within the mean-field approximation, one finds

$$
\begin{aligned}
H_{1}= & \sum_{\mathbf{k}} \Delta_{0}\left(a_{\mathbf{k} \uparrow}^{\dagger} a_{-\mathbf{k} \downarrow}^{\dagger}+b_{\mathbf{k} \uparrow}^{\dagger} b_{-\mathbf{k} \downarrow}^{\dagger}\right) \\
& +\Delta_{1}(\mathbf{k})\left(a_{\mathbf{k} \uparrow}^{\dagger} b_{-\mathbf{k} \downarrow}^{\dagger}-a_{\mathbf{k} \uparrow}^{\dagger} b_{-\mathbf{k} \downarrow}^{\dagger}\right)+\text { H.c. },
\end{aligned}
$$

where the two components of the order parameter are defined as

$$
\begin{aligned}
\Delta_{0} & =\frac{V_{o n}}{N} \sum_{\mathbf{k}^{\prime}}\left\langle a_{-\mathbf{k}^{\prime} \downarrow} a_{\mathbf{k}^{\prime} \uparrow}\right\rangle=\frac{V_{o n}}{N} \sum_{\mathbf{k}^{\prime}}\left\langle b_{-\mathbf{k}^{\prime} \downarrow} b_{\mathbf{k}^{\prime} \uparrow}\right\rangle, \\
\Delta_{1}(\mathbf{k}) & =\frac{V_{n n}}{2 N} \sum_{\mathbf{k}^{\prime}} \gamma_{\mathbf{k}-\mathbf{k}^{\prime}}\left\langle b_{-\mathbf{k}^{\prime} \downarrow} a_{\mathbf{k}^{\prime} \uparrow}-b_{-\mathbf{k}^{\prime} \uparrow} a_{\mathbf{k}^{\prime} \downarrow}\right\rangle .
\end{aligned}
$$

While the onsite order parameter $\Delta_{0}$ is manifestly $\mathbf{k}$ independent, the presence of an anisotropic intersite component $\Delta_{1}(\mathbf{k})$ opens the possibility of unconventional superconductivity. In particular, it is possible to factorize $\Delta_{1}(\mathbf{k})$ in terms of the basis functions of the irreducible representations (irreps) of the point group $D_{6 h}$ as

$$
\Delta_{1}(\mathbf{k})=\sum_{\ell=0}^{2} \Delta_{1 \ell}\left[\phi_{\ell}(\mathbf{k})+i \psi_{\ell}(\mathbf{k})\right]
$$

where $\phi_{0}(\mathbf{k})+i \psi_{0}(\mathbf{k})=\gamma_{\mathbf{k}} / \sqrt{3}$ and $\phi_{\ell}(\mathbf{k})+i \psi_{\ell}(\mathbf{k})=$ $e^{i \mathbf{k} \cdot \delta_{\ell}}-\gamma_{\mathbf{k}} / 3(\ell=1,2,3)$ 31. Their subsets $\left\{\phi_{0}\right\},\left\{\psi_{0}\right\}$, $\left\{\phi_{1}, \phi_{2}\right\},\left\{\psi_{1}, \psi_{2}\right\}$ form a basis for the irreps $A_{1 g}, B_{1 u}$, $E_{2 g}$, and $E_{1 u}$, respectively. One finds that the basis functions of both one-dimensional irreps vanish at the Dirac points $\mathbf{K}=\frac{2 \pi}{3 a}\left(1, \frac{\sqrt{3}}{3}\right), \mathbf{K}^{\prime}=\frac{2 \pi}{3 a}\left(1,-\frac{\sqrt{3}}{3}\right)$. Following standard nomenclature [7], a nonvanishing component $\Delta_{0}, \Delta_{01}$, and $\Delta_{0 \ell}(\ell=1,2)$ will be termed $s$-wave, extended $s$ wave, and $d$-wave, respectively. We have analyzed the gap equations (2) as a function of temperature and chemical potential, as well as of the coupling constants. As is well known, when several competing symmetries are available for the order parameter, the gap equations factorize at $T_{c}$, and symmetry mixing is only possible below $T_{c}[32$. Since the system is actually two-dimensional, the meanfield $T_{c}$ only provides an upper bound for the BerezinskiiKosterlitz-Thouless transition 33. Fig. 1 shows the lowtemperature mean-field phase diagrams for graphene, at various dopings, as a function of the coupling strengths. Solid lines separating filled regions are defined as the locus in the plane of reduced coupling constants, $\left(V_{o n} / t, V_{n n} / t\right)$, such that the system exhibits SC with the relatively low critical temperature $T_{c}=4 \mathrm{~K}$. The limiting case of pristine graphene $(\mu=0$, Fig. 1, upper left panel) requires relatively high coupling strengths in order to develop SC even with a critical temperature as low as $T_{c}=4 \mathrm{~K}$. At $\mu=0$, as $T \rightarrow 0$, one recover the phase diagram of Ref. 22], where however only the $s$ - and $p+i p$-waves symmetries have been addressed. When $\mu \neq 0$, one finds that $T_{c} \rightarrow 0$ when $V_{\text {on }}, V_{n n} \rightarrow 0^{-}$, in agreement with results of Refs. 22, 34, 35. One then needs finite doping in order to have SC at moderately low coupling strengths (Fig. 1, upper right panel, $\mu=0.5 t$ ). Upon doping, $d$-wave symmetry prevails at $T_{c}$ close to the Van Hove singularities in both the valence and conduction bands, while $s$ - or extended $s$-wave symmetry wins out close to the band edges. This is a generic effect of the proximity to an ETT, and has been described as a feature of high- $T_{c}$ superconductivity in the cuprates [36]. At exactly the ETT within the conduction band (Fig. 1, lower left panel, $\mu=1.07 t$ ), $d$-wave symmetry is maximally favored. It has been suggested [5] that fine-tuning the gate voltage in suspended graphene may achieve dopings of practical use. In particular, this may be even easier in uniaxially strained graphene, where the energy slope of the DOS increases with increasing strain [37. Upon further increasing the chemical potential (Fig. 11 lower right panel, $\mu=1.5 t$ ), the pure $d$-wave $\mathrm{SC}$ region shrinks, and eventually gets suppressed altogether. Assuming $V_{\text {on }}=0\left(\Delta_{0}=0\right)$, and neglecting band asymmetry $(s=0)$, one recovers the results of [6, 7]. In particular, $V_{n n}=-2.4 t$ corresponds to Baskaran's proposal of resonating bond ordering in graphite [8].

\section{Single impurity effects}

We next include the effect of a nonmagnetic localized impurity, whose potential is given by

$$
V=a^{2} U_{0} \sum_{\sigma} \Psi_{\sigma}^{\dagger}(\mathbf{x}) \Psi_{\sigma}(\mathbf{x})
$$

where $\Psi_{\sigma}(\mathbf{x})$ is a field operator at position $\mathbf{x}$ with spin projection $\sigma$, and the energy $U_{0}$ measures the impurity potential strength. In terms of the Nambu spinor $\varphi_{\mathbf{k}}^{\dagger}=$ $\left(a_{\mathbf{k} \uparrow}^{\dagger}, b_{\mathbf{k} \uparrow}^{\dagger}, a_{-\mathbf{k} \downarrow}, b_{-\mathbf{k} \downarrow}\right)$, the imaginary time Green's function is defined as $\mathcal{G}\left(\mathbf{k}, \mathbf{k}^{\prime}, \tau\right)=-\left\langle\varphi_{\mathbf{k}}(\tau) \varphi_{\mathbf{k}^{\prime}}^{\dagger}(0)\right\rangle$, and obeys the Gor'kov matrix equation of motion

$$
\sum_{\mathbf{q}}\left[\left(i \omega_{n}-\mathcal{K}_{\mathbf{k}}\right) \delta_{\mathbf{k q}}-\mathcal{V}(\mathbf{k}, \mathbf{q})\right] \mathcal{G}\left(\mathbf{q}, \mathbf{k}^{\prime}, i \omega_{n}\right)=\delta_{\mathbf{k k}^{\prime}},
$$



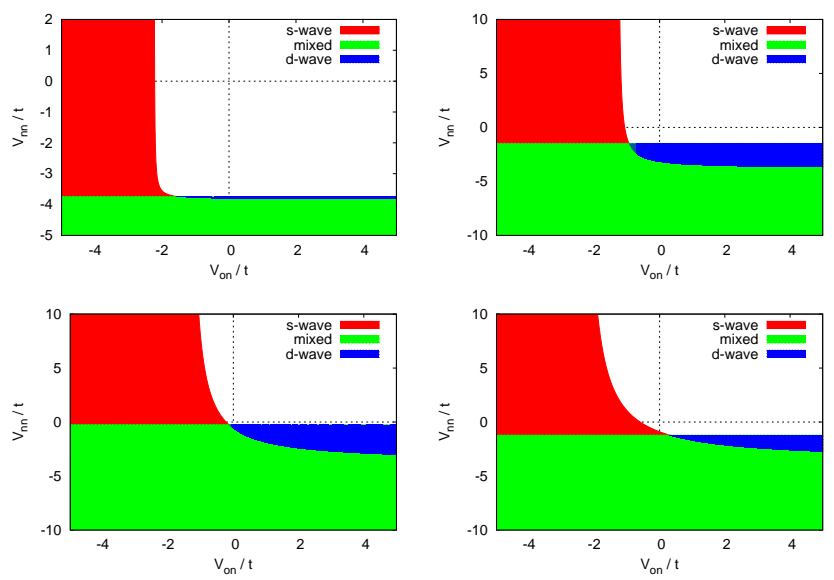

Fig. 1. (Color online) Low-temperature phase diagrams for the symmetry of the SC order parameter as a function of the coupling strengths. Left to right, top to bottom panels show the cases $\mu=0$ (undoped), $0.5 t, 1.07 t$ (Van Hove singularity), and $1.5 t$.

where $\hbar \omega_{n}=(2 n+1) \pi k_{\mathrm{B}} T$ is a fermionic Matsubara frequency. Here, $\mathcal{K}_{\mathbf{k}}$ is a $4 \times 4$ matrix associated with the mean-field SC Hamiltonian,

$$
\mathcal{K}_{\mathbf{k}}=\left(\begin{array}{cc}
K_{\mathbf{k}}^{0} & \Delta_{\mathbf{k}} \\
\Delta_{\mathbf{k}}^{\dagger} & -K_{\mathbf{k}}^{0}
\end{array}\right)
$$

whose $2 \times 2$ blocks are given by

$$
\begin{aligned}
K_{\mathbf{k}}^{0} & =\left(\begin{array}{cc}
\frac{1}{2}\left(E_{\mathbf{k}-}+E_{\mathbf{k}+}\right)-\mu & \left(E_{\mathbf{k}-}-E_{\mathbf{k}+}\right) /\left(2 \gamma_{\mathbf{k}}^{*}\right) \\
\left(E_{\mathbf{k}-}-E_{\mathbf{k}+}\right) /\left(2 \gamma_{\mathbf{k}}\right) & \frac{1}{2}\left(E_{\mathbf{k}-}+E_{\mathbf{k}+}\right)-\mu
\end{array}\right) \\
\Delta_{\mathbf{k}} & =\left(\begin{array}{cc}
\Delta_{0} & \Delta_{1}(\mathbf{k}) \\
\Delta_{1}(-\mathbf{k}) & \Delta_{0}
\end{array}\right)
\end{aligned}
$$

Analogously, the impurity potential enters as a $4 \times 4$ blockdiagonal matrix

$$
\mathcal{V}\left(\mathbf{k}, \mathbf{k}^{\prime}\right)=\left(\begin{array}{cc}
V\left(\mathbf{k}, \mathbf{k}^{\prime}\right) & 0 \\
0 & -V\left(\mathbf{k}, \mathbf{k}^{\prime}\right)
\end{array}\right),
$$

whose $2 \times 2$ blocks have generic elements

$$
V_{\lambda \lambda^{\prime}}\left(\mathbf{k}, \mathbf{k}^{\prime}\right)=a^{2} U_{0} \psi_{\mathbf{k} \lambda}^{*}(\mathbf{x}) \psi_{\mathbf{k}^{\prime} \lambda^{\prime}}(\mathbf{x}),
$$

and $\psi_{\mathbf{k} \lambda}(\mathbf{x})$ is the Bloch wavefunction for sublattice $\lambda=$ $A, B$ employed in the tight-binding diagonalization of the pure sector of the Hamiltonian [38. Eq. (5) is exactly soluble in the SC case, in the absence of impurities, while in the normal case and in the presence of localized impurities at highly-symmetric lattice positions it has been studied in [38. In the general case, its solution can be expressed in terms of an appropriate $T$-matrix as

$$
\begin{aligned}
\mathcal{G}\left(\mathbf{k}, \mathbf{k}^{\prime}, i \omega_{n}\right)= & \delta_{\mathbf{k k}^{\prime}} \mathcal{G}_{0}\left(\mathbf{k}, i \omega_{n}\right) \\
& +\mathcal{G}_{0}\left(\mathbf{k}, i \omega_{n}\right) T\left(\mathbf{x} ; \mathbf{k}, \mathbf{k}^{\prime}, i \omega_{n}\right) \mathcal{G}_{0}\left(\mathbf{k}^{\prime}, i \omega_{n}\right)
\end{aligned}
$$

where $\mathcal{G}_{0}\left(\mathbf{k}, i \omega_{n}\right)$ is the solution of the SC pure case. Fourier transforming back in real space via $\psi_{\mathbf{k} \lambda}(\mathbf{r})$ and performing an analytic continuation to real frequencies, the local density of states at position $\mathbf{r}$ can be expressed as $\rho(\mathbf{r}, \omega)=$ $-\frac{1}{\pi} \operatorname{Im} G(\mathbf{r}, \mathbf{r}, \omega)$. In what follows, we will use Gaussian pseudoatomic wavefunctions $\phi(\mathbf{r})=Z_{g} \exp \left(-Z_{g}^{2} r^{2} / 24 a^{2}\right) /(2 a \sqrt{3 \pi})$ $\left(Z_{g}=11.2\right)$ 38. to expand the Bloch wavefunctions within the tight-binding approximation as $\psi_{\mathbf{k} \lambda}(\mathbf{r})=N^{-1 / 2} \sum_{j} \phi(\mathbf{r}-$ $\left.\mathbf{R}_{j}^{\lambda}\right) e^{i \mathbf{k} \cdot \mathbf{R}_{j}^{\lambda}}$, where $\mathbf{R}_{j}^{\lambda}$ are vectors of the $\lambda=A, B$ sublattices. Placing the impurity on an $A$ site, at $\mathbf{r}=\mathbf{0}$ say, and retaining only the zeroth order approximation, one has $\psi_{\mathbf{k} A}(\mathbf{0}) \approx N^{-1 / 2} \phi(\mathbf{0})$ and $\psi_{\mathbf{k} B}(\mathbf{0}) \approx 0$. In this limit, the impurity potential matrix simplifies to $\mathcal{V}\left(\mathbf{k}, \mathbf{k}^{\prime}\right) \equiv \mathcal{V}_{0}=$ $\phi^{2}(\mathbf{0}) a^{2} U_{0} N^{-1} \sigma_{3} \otimes\left(\sigma_{0}+\sigma_{3}\right) / 2$, where $\sigma_{0}, \sigma_{3}$ are Pauli matrices. Correspondingly, the analytically continued $T$ matrix assumes the $\mathbf{k}$-independent form $T(\mathbf{r}=\mathbf{0} ; \omega)=$ $\mathcal{V}_{0} \mathcal{M}^{-1}(\mathbf{0}, \omega)$, where zeroes of the determinant of $\mathcal{M}(\mathbf{0}, \omega)=$ $1-\sum_{\mathbf{q}} G_{0}(\mathbf{q}, \omega) \mathcal{V}_{0}$ as a function of $\omega$ are connected with impurity-induced bound states or well-defined resonances.

Fig. 2 shows the LDOS on an atomic site $(\mathbf{r}=\mathbf{0})$ for pure SC graphene $\left(U_{0}=0\right)$. Here, and in what follows, we shall consider slightly doped graphene $(\mu=0.5 t)$, in order to stabilize the SC phase without requiring exceedingly high coupling strengths. This corresponds to having the Fermi surface in the shape of two disconnected rings, centered around either inequivalent Dirac point. In the $s$-wave case (Fig. 2 top panel), the LDOS assumes a typical BCS-like shape, with well-defined coherence peaks located at $\omega \approx \pm \Delta_{0} / 2$, and no spectral weight is available within the gap. Away from the gapped region, the LDOS is not affected by superconductivity, and in particular the Van Hove singularities remain intact. Qualitatively similar results have been obtained in the case of an extended $s$-wave order parameter. This is because, despite the nontrivial k-dependence, the order parameter vanishes precisely at the Dirac points, viz. where the bands vanish if $\mu=0$. On the other hand, in the $d$-wave case (Fig. 2, bottom panel), the onsite LDOS vanishes linearly at $\omega=0$, and the coherence peaks are considerably reduced. This is typical of unconventional superconductors, and is in fact a hallmark of $d$-wave superconductivity in the cuprates [27. In Fig. 2, we have assumed in-phase $d$-wave components for the order parameter, i.e. $\Delta_{11}=\Delta_{12}$. Other phase relations are possible, such as $\Delta_{12}=\Delta_{11} e^{-i \pi / 3}$. In the latter case, $\Delta_{1}(\mathbf{k})$ vanishes only at the Dirac points. Therefore, in the case of doped graphene, the SC order parameter has no node along the Fermi surface, and one finds a fully gapped LDOS, qualitatively similar to the $s$-wave case. This is in agreement with the results of [7]. However, while an out-of-phase order parameter is numerically favored in the case of pure $d$-wave symmetry, we find that a nonzero $s$-wave component stabilizes in-phase $d$-wave superconductivity.

We consider now the effect of a single, localized, nonmagnetic impurity, located on a lattice site. In the normal state, we recover the results of [38] for a site-like impurity. Fig. 3 shows our results for the LDOS in the SC state. In the $s$-wave case (Fig. 3, top panel), the onsite LDOS is characterized by the opening of a gap and the formation of well-defined coherence peaks, while the Van Hove sin- 

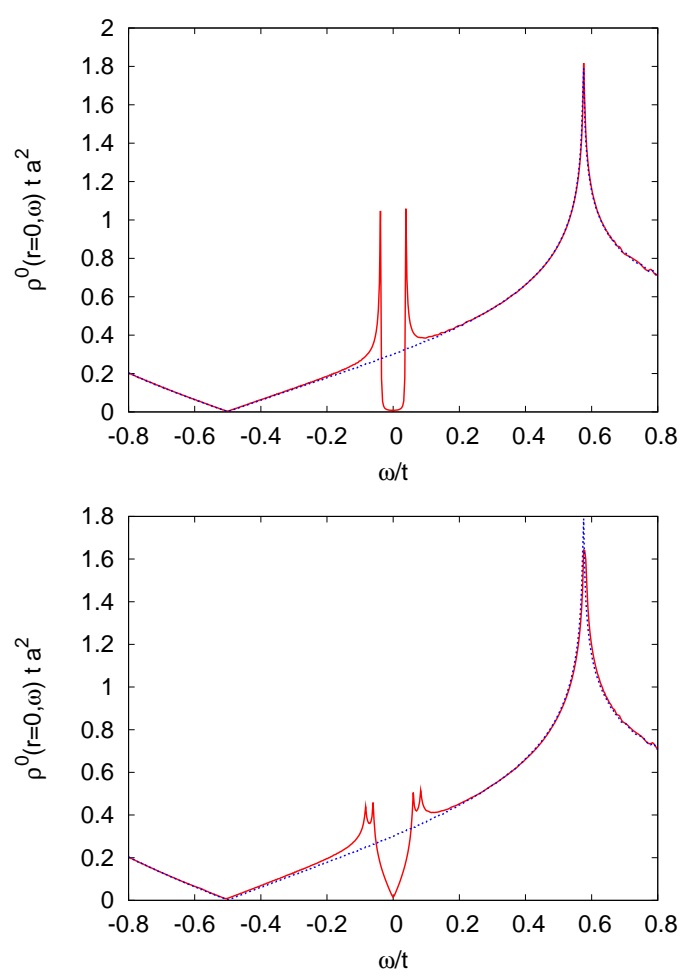

Fig. 2. (Color online) LDOS for pure SC (solid line) and normal-state graphene (dotted line). Top panel refers to $s$-wave symmetry, while bottom panel refers to $d$-wave symmetry, with in-phase components of the order parameter. In both cases, $\mu=0.5 t$ and $U_{0}=0$.

gularity gets suppressed, as in the normal state. On the other hand, the LDOS on a nearest-neighbor site gets enhanced with respect to its normal state counterpart, and the Van Hove singularities undergo no significant reduction. More importantly, by analyzing the determinant of the matrix $\mathcal{M}(\mathbf{r}, \omega)$, one finds that no bound state is possible within the gapped region in the $s$-wave case. In the $d$-wave case (Fig. 3, bottom panel), while a similar analysis applies as in the $s$-wave case at large energies, the situation is completely different within the gapped region. One finds that sufficiently high impurity coupling strengths $\left(-3.8 t \lesssim U_{0} \lesssim-0.8 t\right.$, in the case under study) allow the formation of well-defined bound state pairs within the gapped region. (That bound states should come in pairs follows from the particle-hole mixing, characteristic of the SC state 27.) This is indeed apparent from Fig. 33(bottom panel), with the appearance of sharp peaks in the onsite LDOS. Such a result is similar to the $d$-wave SC state of the cuprates, and has indeed been verified experimentally in STM experiments in Bi2212 [24,25].

\section{Conclusions}

In summary, we derived the possible symmetries of a SC order parameter compatible with the honeycomb lattice structure of graphene. We discussed the SC phase diagram at low-temperature as a function of doping and interaction
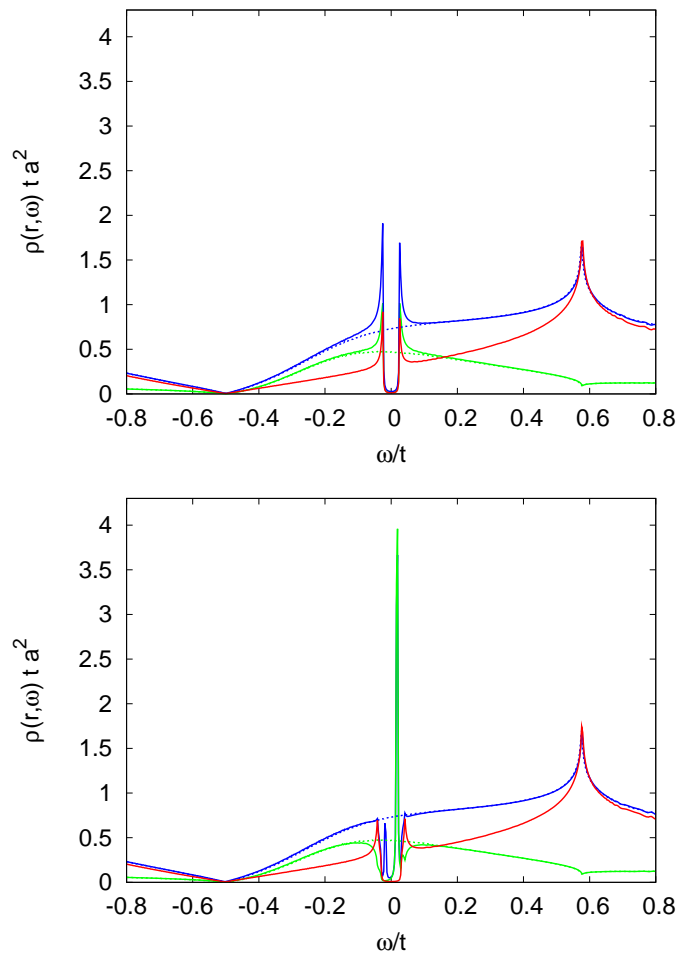

Fig. 3. (Color online) LDOS in the presence of a localized impurity for SC (solid lines) and normal-state graphene (dotted lines). Green lines refer to LDOS on top of the impurity $(\mathbf{x}=$ $\mathbf{0})$, while blue lines refer to LDOS on a nearest-neighbor site $\left(\mathbf{x}=\delta_{3}\right)$. Red lines refer to the on-site $\operatorname{LDOS}(\mathbf{x}=\mathbf{0})$ in the SC phase, in the absence of impurities. Top panel refers to extended $s$-wave symmetry, while bottom panel refers to $d$-wave symmetry. In both cases, $\mu=0.5 t$ and $U_{0}=-0.75 t$.

strengths. In particular, we showed that unconventional pairing may stabilize close to an ETT, with possible admixtures of a subdominant $s$-wave contributions.

We then evaluated the LDOS around an isolated impurity in the SC phase, and suggested that STM experiments may detect the occurrence of unconventional pairing in intrinsically or proximity-induced superconducting graphene. This will be helpful in determining the pairing mechanism of superconducting graphene. We have limited our study to isolated nonmagnetic impurities. Such a condition may be realized in the limit of low impurity concentration, where it is safe to neglect impurity effects on the overall transport properties of graphene.

\section{References}

1. K.S. Novoselov, D. Jiang, F. Schedin, T.J. Booth, V.V. Khotkevich, S.V. Morozov, A.K. Geim, Proc. Nat. Acad. Sci. 102, 10451 (2005)

2. A.H. Castro Neto, F. Guinea, N.M.R. Peres, K.S. Novoselov, A.K. Geim, Rev. Mod. Phys. 81, 000109 (2009)

3. C. Honerkamp, Phys. Rev. Lett. 100, 146404 (2008)

4. J. González, F. Guinea, M.A.H. Vozmediano, Phys. Rev. B 63, 134421 (2001) 
5. J. González, Phys. Rev. B 78, 205431 (2008)

6. A.M. Black-Schaffer, S. Doniach, Phys. Rev. B 75, 134512 (2007)

7. Y. Jiang, D.X. Yao, E.W. Carlson, H.D. Chen, J.P. Hu, Phys. Rev. B 77, 235420 (2008)

8. G. Baskaran, Phys. Rev. B 65, 212505 (2002)

9. H.B. Heersche, P. Jarillo-Herrero, J.B. Oostinga, L.M.K. Vandersypen, A.F. Morpurgo, Nature 446, 56 (2007)

10. Y. Kopelevich, P. Esquinazi, J.H.S. Torres, S. Moehlecke, J. Low Temp. Phys. 119, 691 (2000)

11. M.S. Dresselhaus, G. Dresselhaus, Adv. Phys. 51, 1 (2002)

12. G. Csányi, P.B. Littlewood, A.H. Nevidomskyy, C.J. Pickard, B.D. Simons, Nat. Phys. 1, 42 (2005)

13. T. Weller, M. Ellerby, S.S. Saxena, R. Smith, N. Skipper, Nature Phys. 1, 39 (2005)

14. O. Gunnarsson, Rev. Mod. Phys. 69, 575 (1997)

15. M. Calandra, F. Mauri, Phys. Rev. B 74(9), 094507 (2006)

16. J.S. Kim, L. Boeri, R.K. Kremer, F.S. Razavi, Phys. Rev. B 74, 214513 (2006)

17. L. Boeri, G.B. Bachelet, M. Giantomassi, O.K. Andersen, Phys. Rev. B 76(6), 064510 (2007)

18. A. Sanna, G. Profeta, A. Floris, A. Marini, E.K.U. Gross, S. Massidda, Phys. Rev. B 75(2), 020511 (2007)

19. A.Y. Liu, I.I. Mazin, Phys. Rev. B 75(6), 064510 (2007)

20. A. Grüneis, C. Attaccalite, A. Rubio, D.V. Vyalikh, S.L. Molodtsov, J. Fink, R. Follath, W. Eberhardt, B. Büchner, T. Pichler, Phys. Rev. B 79(20), 205106 (2009)

21. T. Valla, J. Camacho, Z.H. Pan, A.V. Fedorov, A.C. Walters, C.A. Howard, M. Ellerby, Phys. Rev. Lett. 102(10), 107007 (2009)

22. B. Uchoa, A.H. Castro Neto, Phys. Rev. Lett. 98, 146801 (2007)

23. T.O. Wehling, H.P. Dahal, A.I. Lichtenstein, A.V. Balatsky, Phys. Rev. B 78, 035414 (2008)

24. E.W. Hudson, S.H. Pan, A.K. Gupta, K.W. Ng, J.C. Davis, Science 285, 88 (1999)

25. S.H. Pan, E.W. Hudson, K.M. Lang, H. Eisaki, S. Uchida, J.C. Davis, Nature (London) 403, 746 (2000)

26. S. Chakravarty, R.B. Laughlin, D.K. Morr, C. Nayak, Phys. Rev. B 63, 094503 (2001)

27. D.K. Morr, Phys. Rev. Lett. 89, 106401 (2002)

28. N. Andrenacci, G.G.N. Angilella, H. Beck, R. Pucci, Phys. Rev. B 70, 024507 (2004)

29. R. Saito, M. Fujita, G. Dresselhaus, M.S. Dresselhaus, Physical properties of carbon nanotubes (Imperial College Press, London, 1998)

30. S. Reich, J. Maultzsch, C. Thomsen, P. Ordejón, Phys. Rev. B 66, 035412 (2002)

31. F.M.D. Pellegrino, G.G.N. Angilella, in preparation ..., ... (2010)

32. G.G.N. Angilella, R. Pucci, F. Siringo, A. Sudbø, Phys. Rev. B 59, 1339 (1999)

33. K. Fossheim, A. Sudbø, Superconductivity. Physics and Applications (J. Wiley and Sons, Chichester, 2004)

34. B. Uchoa, G.G. Cabrera, A.H. Castro Neto, Phys. Rev. B 71(18), 184509 (2005)

35. E. Zhao, A. Paramekanti, Phys. Rev. Lett. 97(23), 230404 (2006)

36. G.G.N. Angilella, E. Piegari, A.A. Varlamov, Phys. Rev. B 66, 014501 (2002)

37. F.M.D. Pellegrino, G.G.N. Angilella, R. Pucci, Phys. Rev. B 81, 035411 (2010)

38. F.M.D. Pellegrino, G.G.N. Angilella, R. Pucci, Phys. Rev. B 80, 094203 (2009) 